\title{
Development of Multi-functional Electronic Power Assisting Device of Substation Equipment
}

\author{
Yi Yongfei ${ }^{1, a}$, Zhu Yuanda ${ }^{1, a}$, Yang Ze ${ }^{2, b}$, Wang Wei ${ }^{1, a}$, Jin Xin ${ }^{1, a}$ \\ ${ }^{1}$ Liaoning Skill Training Center \\ ${ }^{2}$ Benxi Power Supply Company, State Grid Liaoning Electric Power Supply Co., Ltd. \\ a22181221@qq.com, b bxwl_650928@126.com
}

Keywords: speed regulation, positive and reverse rotation

\begin{abstract}
The direct-current motor has been widely used in the modern electronic products, such as automatic control system, electronic equipment, home appliances, and electronic toys. The tape recorder, record player, recorder, and computer cannot work without the direct-current motor. So the control of the direct-current motor is a very practical technology. The direct-current motor has good starting performance and speed regulation characteristics, which is characterized by the big starting torque, big maximum torque, the smooth and economical speed regulation in a wide range, easy rotational speed regulation and high efficiency after speed regulation. Compared with AC speed regulation, the direct-current motor is complex in structure, with high production cost and heavy maintenance work. The direct-current regulator is widely applied in CNC machine tools, paper printing, textile printing and dyeing, fiber optic cable equipment, packaging machinery, electrical machinery, food processing machinery, rubber machinery, biological equipment, printed circuit board equipment, experimental equipment, welding and cutting, light industry machinery, logistics and transportation equipment, medical equipment, communication equipment, radar equipment, satellite ground receiving system and other industries. This paper aims at a hand-held speed regulation device, which can make the manual drive of substation maintenance to electric drive, so as to save energy and time.
\end{abstract}

\section{Introduction}

In daily life and production, the direct-current motor is often used, but the intelligent control of the direct-current motor speed has become a growing concern. For this, starting from the principle of the device, and focusing on the hardware circuit, this paper designs a digital direct-current motor speed regulation system, which includes the controller, pulse width modulation system, speed detection device, display, and keys. It takes the AT89C52 microcontroller as the central controller, the photoelectric sensor as the frequency detection device, with the pulse width of MAX5441 - D/A to do regulation, and then to control motor speed, which realizes variable PWM control motor speed, achieving the engineering index, and realizing the intelligent control of the motor.

\section{Working principle}

The digital direct-current motor speed regulation system has five parts, including the controller, the PWM driver, the speed detecting circuit, the key setting, and the display output, in which PWM driver, speed detection circuit, key setting, display output are controlled by the controller.

The working principle of direct-current speed regulator is briefly introduced as follows:

The direct-current speed regulator is the equipment of regulating the direct-current motor speed. Its upper end is connected to AC power supply, while the lower end is connected to the direct-current motor. The direct-current speed regulator changes alternating current into direct-current of two outputs, one of which inputs to the direct-current motor excitation (stator) and the other to the direct-current motor armature (rotor) and the direct-current speed regulator adjusts the rotate speed of the direct-current motor by controlling the armature direct-current voltage. At the same time, the direct-current motor gives a feedback current to the speed regulator, which can 
judge the rotate speed of the direct-current motor according to the feedback current and modify the voltage output of armature so as to reregulate the rotate speed of motor.

\section{Implementation plan}

The speed regulation scheme of the direct-current motor generally has the following three ways: 1 , to change the armature voltage; 2, to change the excitation winding voltage; 3 , to change the armature circuit resistance. The most commonly used one is to adjust the speed regulation system, namely, 1 (to change the armature voltage).

A modular speed regulator of direct-current motor combines the power supply, control and driving circuit together, and uses three-dimensional structure layout. Its control circuit uses micro power components, the isolation and conversion of current and voltage are achieved by a photoelectric coupler, its circuit ratio constant, integral constant and differential constant use PID adapter to do adjustment. The speed regulator is small and light, can be used alone or directly mounted on the direct-current motor to form an integrated direct-current motor of speed regulation, which have all the functions of the speed regulator.

The rapid development of modern industrial automation provides a wide range of applications and updates to the control and speed regulation of the direct-current motor, such as Remote signal transmission, remote speed regulation, remote speed regulation and control in high temperature environment, and manual automatic integration.

The rotate speed of the motor is detected by photoelectric sensor and the trigger pulse is generated and counted by the single chip triggered subharmonically by the trigger and. The standard value is got by the timer counter and the calculation and process of data by single chip builds the relation between time and pulse, and therefore the rotate speed of the motor per minute is obtained. The rotate speed subtracts the given speed is the deviation, which is then adjusted by PID, so that the speed reaches the given speed and realizes speed control. The speed regulation of the motor is controlled by PWM, which is composed by generating circuit of triangular wave, comparator, and feedback circuit. The closed feedback control is realized when the single chip inputs deviation value to the high speed DA, which outputs feedback value to PWM. The motor is driven by integrated $\mathrm{H}$ bridge chip, and in order to prevent the voltage disturbance, the control system is added level electric current of filter circuit. The positive and reverse rotation of the motor is controlled by the positive and negative direction of the voltage. When direction switches, the filter circuit inductance of the circuit will produce anti electromotive force, affecting the power supply system, and even making the system unable to work normally. So the relay is picked as the change-over switch. The rotate speed can be set by keys, to control the positive or reverse direction of the motor. Display circuit uses the bright digital tube display. The system is designed based on the principle of the coordinated high precision and real time.

By changing the duty cycle of the output square wave, the average current of the load is changed from the $0-100 \%$, which changes the load, the light intensity and the motor speed. The pulse width modulation (PWM) mode realizes the regulation of light and speed. Its advantage is that the energy power of electricity can be fully utilized, and the efficiency of the circuit is high. For example: when the output gets the square wave pulse of $50 \%$, the output power of PWM circuit is also $50 \%$, that is, almost all of the energy is converted to load. When common resistance is used to reduce voltage and regulate speed, to make the load obtain the maximum power of $50 \%$, power supply must provide more than $71 \%$ of the output power, $21 \%$ of which is consumed in lowing voltage of resistance and heat consumption. Since most of the energy is consumed in the resistance, the remaining is the output energy, and the conversion efficiency is very low. In addition, the HW-A-1020 type takes use of the switch mode which barely has any heat consumption. It has very large torque at low speed because the speed regulator has auto tracker of PWM. Besides it adopts pulse width modulation (PWM) mode, which makes the working load get almost full power supply voltage, which is helpful to overcome the coil resistance inside the motor and make the motor produce a greater torque rate. 
HW-A-1020 type speed regulation can,because the speed regulator with automatic tracking PWM, in addition to the use of

Rotate speed detection works in the field, so the accuracy of measurement and its anti-interference property is the foundation of the whole system design. The photoelectric sensor of LTH-301A is selected, which is fast and has high reliability and anti magnetic interference property. The sensor generates a low pulse each time when it is subject to light. The motor speed is calculated, by counting the clock cycles of the single chip of a low pulse period.

Driving motor is in need of sufficient current. In the design, the integrated H bridge of L293D, L293D driver of 1.2A, and embedded integrated $\mathrm{H}$ bridge compose the push-pull circuit. L293D receives the two sets of drives composed by PWM voltage, which can be used for alternatively and easy to control the rotation direction of the motor. LC filter circuit is added to the system, to filter the ripple of circuit, and eliminate the interference of the circuit disturbance to single chip and at the same time, the relay is added to control the positive reverse rotation to prevent the counter electromotive force generated during switchover affects the working of the system. Through a series of protective measures, the stable speed regulation of the motor can be ensured.

After circuit is connected, the connection between power and ground should be checked see whether it is correct. Otherwise, it is easy to burn chip circuit, so the circuit should not be switched on until carefully check and confirmation. First of all, the generating circuit of the sawtooth wave circuit is tested to see whether it produces sawtooth wave, and then the constant current flow source current and LM358 positive input voltage are adjusted, and the approximate frequency and amplitude are got. And then it is debugged combined with the software. L293D motor drive circuit is detected, whose two control terminals EN controls a set of bridge circuit respectively and each time only a set is effective. Their working condition should be checked separately. Photoelectric sensor is a part of direct testing motor, and it should be carefully examined, and its waveform should be taken by the oscilloscope to verify whether the waveform is a pulse wave. The high level reaches $5 \mathrm{~V}$, and the low level is less than $1 \mathrm{~V}$. When there is no error through debugging, it is powered on and the next step of the software debugging is to be done.

The packing of the motor speed regulation system includes digital display, keys, motor and detection device. The left key is for setting and exiting, when setting the state, the digital tube tracks and displays the set value. While setting, the middle key is for adding and pressing the set key (left), add can be realized. While setting, the right key is for subtracting, and pressing the setting key (right), subtraction can be realized. Exiting set is the control state. In control state, pressing the right key, the reverse rotational speed is realized. When the upper indicator light is on and the lower indicator is off, it is the control state, and when all the lights are on, it is the control state.

In the joint position of the motor rotor, the substation maintenance of the side manual rocker connector at a time is able to realize the manual operation of the switch, which greatly saves time and manpower.

\section{Points for attention}

(1) before usage, the power cord has to be checked whether it is damaged. If it is, it must be wrapped by insulation tape. During usage, it should not be immersed in water and not contact the heat source or corrosive media.

(2) the protective grounding (grounding zero) must be applied.

(3) before usage, the switch must be in the off state, and the sudden rotation must be avoided while it is being plugged into the power outlet.

(4) before usage, it should be raced for 0.5 to $1 \mathrm{~min}$ and be checked to see whether the rotate parts is flexible, whether there is abnormal noise, whether screws loosen, and whether the commutator spark is normal.

(5) during usage, the electric drill should not be gripped by a single hand but both hands, and the correct operating posture should be adopted.

(6) the gapped drill cannot be used. While drilling, the downward pressure should not be too big, so as to prevent break of the drill. 
(7) such acts as replacing connector should be carried out while the power is disconnected.

(8) the small work pieces must be clamped by jags and then used.

(9) the operating strength should not be too big, to prevent the connector flying out to hurt people.

(10) before the operation, the connector should be carefully examined to see whether there is a crack or damage. If there is, it should be replaced immediately.

(11) the direction of rotation and feeding should be noted.

(12) the power should be cut off at first, and when it completely stops, the work pieces are taken from the tool.

\section{Conclusion}

Infinitely variable speed continuous current dynamo and transmission gearing are designed in accordance with the positive and reverse rotation theory of electrical Machinery towage, thus possessing the functions of forward, stop and reversal and meeting working demands with its switch from high, intermediate and low speed. Therefore, it can enhance working efficiency and guarantee the overhaul quality in actual work. At the same time, it can also offer switching off, switching on, forward rotation and reverse rotation of the equipment.

\section{Reference}

[1] Zhai Yuwen, Liang Wei, Ai Xuezhong, Shi Yungui. Electric design and implementation. China Power Press.2005.

[2] Deng Xingzhong. The control of mechanical and electrical transmission. Huazhong University of Science and Technology Press.2004.

[3] Zhang Yigang. The principle and application of single chip microcomputer. Beijing: Higher Education Press.2004.

[4] Chen Runtai, Xu Kun. Detection technology and intelligent instrument. Hunan: Central South University Press.2002.

[5] Zhang Jin, Ji Shengchang, Shen Qi, et al. Study on $35 \mathrm{kV}$ insulator string flashover voltage washed by charged water vapor[J]. High voltage apparatus, 2010, 46 (7):61.65. 